\title{
Local Rigidity as a Criterion of Gas Permeation of Polymer and Composition Materials; PAL and TSL Experiments
}

\author{
V.P. Shantarovich ${ }^{a, *}$, V.W. Gustov ${ }^{a}$, E.V. Belousova ${ }^{a}$, A.V. Polyakova ${ }^{a}$, \\ V.G. BEKESHEV ${ }^{a}$, I.B. KEVDINA ${ }^{a}$, YU.P. YAMPOLSKII ${ }^{b}$ AND A.V. PASTUKHOV ${ }^{c}$ \\ ${ }^{a}$ Semenov Institute of Chemical Physics, Russian Academy of Sciences, Kosygina 4, Moscow, Russia \\ ${ }^{b}$ Topchiev Institute of Petrochemical Synthesis, Russian Academy of Sciences \\ Leninskii Prospect 29, Moscow 119991, Russia \\ ${ }^{c}$ Nesmeyanov Institute of Organoelement Compounds, Russian Academy of Sciences, Vavilova 28, Moscow

\begin{abstract}
Various commercial and industrial properties of polymeric materials can be developed by the way of purposeful synthesis. For example, there are polymeric materials of extremely developed intrinsic microporosity. In attempts to further develop membrane properties (permeability and selectivity), the researchers artificially increase the pore sizes by various external influences, such as plastification by saturation of polymers with gases, sometimes in supercritical state, filling of a polymer with some non-organic components (zeolites). Sometimes, porosity of the composition appears to become higher than that of the components. This porosity, in difference with intrinsic one, can be called externally affected. The reason of these phenomena is not always clear, and in order to get some ideas in this field we suggest an experimental study using a combination of the two genetically interconnected (by spur processes) methods: positron annihilation lifetime spectroscopy and thermostimulated luminescence. This paper summarizes recently published and completely new results of the authors in order to illustrate the benefits of this experimental approach.
\end{abstract}

DOI: $10.12693 /$ APhysPolA.125.806

PACS: 61.41.+e, 61.43.Fs, 68.43.Vx, 78.70Bj, 82.35.Lr

\section{Introduction}

Positron annihilation lifetime (PAL) measurements were used for years for the studies of free volume in polymers [1-4]. We continue this direction on example of membranes and sorbents.

One of the specific parts of the investigation includes modified materials. They are modified in a way to obtain new unique properties. For example, better permeability or selectivity in membrane materials, more stable operation properties of heterophase polymer compositions. It was shown previously that for membranes, the promising way is a creation of compositions of polymers and zeolites or some other admixtures (mixed matrix membranes, MMMs) [5-7]. Another way is a saturation of a polymer with gas in a supercritical state with following increase of temperature [8].

The second specific feature of this study is a combination of the data of two experimental methods: PAL measurements and thermostimulated luminescence (TSL). The last one is the emission of energy (light) by a substance on heating, after preliminary irradiation at low temperature. As it was stressed recently, both methods are genetically interconnected by the same radiation-chemical nature of the process (spur process). At low temperatures, intensity of the positronium formation and

*corresponding author; e-mail: shant@center.chph.ras.ru integral intensity of TSL are dependent on the storage of the spur electrons (weakly bound electrons). The obtained results make another proof of the spur mechanism of positronium formation [9].

In this study, we wanted to check the benefits of combination of the room temperature PAL data and low temperature TSL data for investigation of some new membrane materials and heteropolymers. The logic of interpretation was as follows. Polymer membrane permeation for some gases depends on the local free volume. This elementary free volume determines the ortho-positronium (o-Ps) lifetime, too. Further on, there is a theoretical suggestion that the free volume (and corresponding long-lived o-Ps component) depends on the polymer local rigidity [10]. In its turn, the rigidity determines the shape of the curve of the integral intensity of the TSL. The shape is characterized in our case by relative intensity of the high temperature (above 200-250 K) component (so-called parameter " $\alpha$ ") [9].

In this paper, the correlation is illustrated by the data on the PAL, TSL and permeation parameters for a number of porous and novel membrane materials, such as cross-linked polystyrenes, poly (3-methylsilyl propine) PTMSP [3], polymer of intrinsic microporosity PIM-1 (polybenzodioxane) [3, 11], perfluoro-polymers AF1600 and AF2400 [3], and mixed matrix membranes MMMs composed of metal organic frameworks MOF (SAPO or ZIF [6]) and polymers PIM or poly(hexafluoro propylene) (PHFP). The obtained results confirm the idea on the interconnection between the permeability, free volume, and local rigidity (local tensions, steric hindrances) 
in polymer matrix. This makes it possible to conclude sometimes about membrane properties even without permeation measurements on the bases of PAL and/or TSL data. In this discussion of PAL and TSL data, we distinguish however intrinsic free volumes for homogeneous glassy polymers and externally affected or created free volumes in compositions.

\section{Low temperature bleaching effect for TSL and Ps formation}

It is known that positron annihilation lifetime measurements are useful for the studies of effective sizes of elementary free volume (EFV) and their distribution in a polymer [4]. This approach stems from the fact that $o$-Ps atom, the bound state of positron and electron in the ortho- or triplet state tends to localize in the EFV and $o$-Ps lifetime depends on the EFV effective size and shape. Some information can be derived on the number density of the EFV, too, but obtaining this information is more complicated since intensity of the $o$-Ps component, the parameter, interconnected with the number density, depends not only on the number of EFV, but also on the concentration of electrons in the positron spur [12]. The last dependence was confirmed recently by experiments on the low temperature positronium formation, where additional amount of positronium was formed due to the storage of the shallow trapped (weakly bound) electrons, when the sample was irradiated $[13-15]$. On the other hand, it is known that the same weakly bound electrons are responsible for TSL [16]. Interconnection of the effects of low temperature positronium formation and TSL was confirmed by bleaching of the both effects by light [13-17]. Figure 1 illustrates an example of the dependence of the TSL integral intensity for PE on the time of bleaching. It is also obvious that the character of the TSL curves as well as the dependence of the low temperature positronium formation on temperature (Fig. 2) are the derivatives of the stability of the weakly bound electrons in the traps and, in this way, on the rigidity of a polymer structure.

\section{Advantages of the PAL and TSL data combination}

The combination of TSL (relatively low temperatures) and PAL (room temperature) data are still effective in the studies of polymer structure compositions, multicomponent polymeric materials, when the specific $o$-Ps lifetimes are close enough and unresolved by the PAL spectroscopy. The objects of such experimentation [7] were chosen to be isotactic polypropylene (PP) and its blends with ethylene-propylene-diene terpolymer EPDM (0-100\%), containing the non-vulcanized and the vulcanized rubber phases and the catalyst of vulcanization (CV) partly inhibiting luminescence. The CV was distributed somehow between the microphases. Long-lived $o$-positronium components of PP and EPDM were very

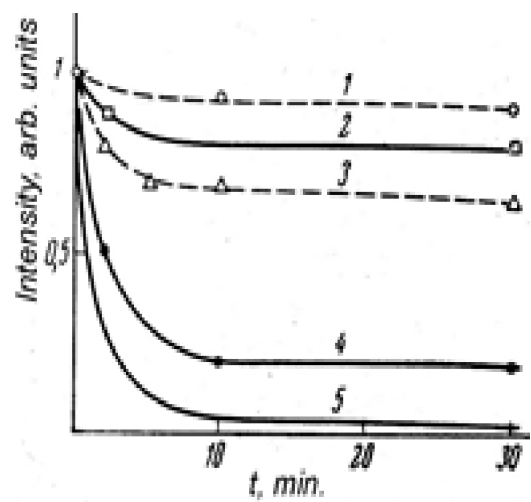

Fig. 1. A dependence of integral intensity of the TSL curve for $\mathrm{PE}$ on the time of bleaching by light of different wave length $(\mathrm{nm}): 1-1000 ; 2-800 ; 3-612$ $4-405 ; 5-\mathrm{UV}[16]$.

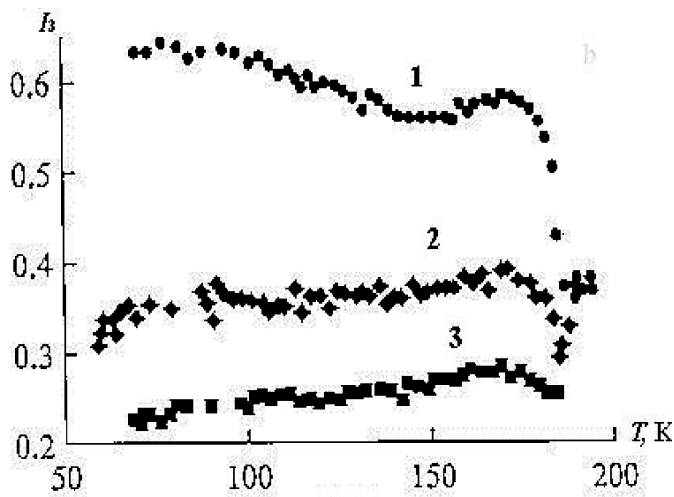

Fig. 2. Inhibition of Ps formation (long-lived component intensity $I_{3}, \%$ ) by the light of various wave lengths in frozen hexane: 1 - in dark, 2 - light, 3 - UV [17] as an evidence of various energies of electron trapping up to several $\mathrm{eV}$.

close, about $2.5 \mathrm{~ns}$ and could not be resolved. However, TSL curves of the components have their individual, specific shapes (Fig. 3) [7]. This enables to get some information on the distribution of the $\mathrm{CV}$ between $\mathrm{PP}$ and EPDM components: inhibition of the PP TSL component more effective than that expected from $\mathrm{PP}$ weight percent in the blend testifies on existence of some part of $\mathrm{CV}$ outside of EPDM, in the PP phase.

\section{Free volume and rigidity of polymer structure}

A number of studies [11, 18-20] had suggested the relation between the free volume and rigidity, stiffness of the polymer chains. Hofmann et al. had published some papers $[18,20]$ on molecular simulation of the EFV distribution in stiff chain polymers, such as poly[(trimethylsilyl)-1-propyne] (PTMSP, $T_{\mathrm{g}}>290^{\circ} \mathrm{C}$ ), where rigidity is related with the presence of the bulky $\mathrm{C}-\mathrm{Si}\left(\mathrm{CH}_{3}\right)_{3}$ moieties in the polymer structure. Rigidity of polymer structure was mentioned also by Budd et al. [11] as the main 


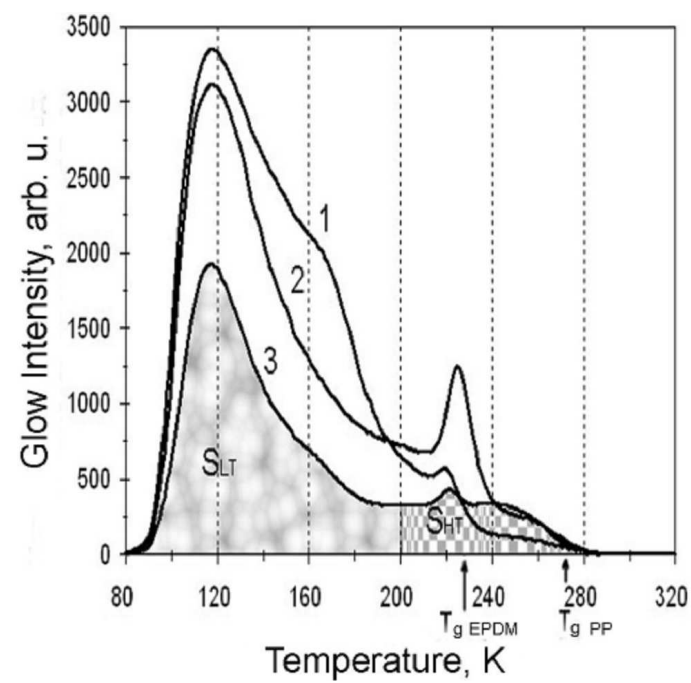

Fig. 3. Fluorescence intensity versus temperature for PP-EPDM blend 50/50: (1) non-vulcanized mixture, (2) vulcanized mixture with $\mathrm{CV}$ concentration $S=1$, (3) vulcanized mixture with $S=3$. Procedure of the parameter $\alpha=S_{\mathrm{HT}} / S_{\mathrm{LT}}$ calculation is shown on example of the sample (3) $[7,9]$.

reason of the high free volume and specific surface in the novel membrane materials of intrinsic microporosity (PIM). For these polymers, there are no single bonds in the backbone about which rotation can occur. However, at intervals along the backbone there are spiral centers (i.e., a single tetrahedral $\mathrm{C}$ atom shared by two rings) which introduces a sharp bend into the chain. The result is an inflexible but contorted polymer molecule. During the film formation or precipitation from solution, sufficient free volume is trapped in the solid state, so that the polymers behave essentially as microporous materials.

Hyper-cross-linked polystyrene networks represent another new class of polymeric materials [19]. They have been obtained by introducing a large number of the bridges between strongly solvated polystyrene chains. Therefore, a very large free volume characterizes dry hyper-cross-linked networks. Indeed, apparent inner surface area achieves $1000-1800 \mathrm{~m}^{2} / \mathrm{g}$ and the pore volume amounts to $0.3-0.5 \mathrm{~cm}^{3} / \mathrm{g}$. Depending on the cross-linker and starting agents the materials were called LPSX, LPSE, CPSX, and CPSE [3, 19].

Finally, among the other glassy membrane materials we shall discuss amorphous teflons (AF), such as AF1600 $\left(T_{\mathrm{g}}=160{ }^{\circ} \mathrm{C}\right)$ and AF2400 $\left(T_{\mathrm{g}}=240^{\circ} \mathrm{C}\right)$, copolymers of tetrafluoroethylene and perfluorodioxolane, containing different amounts of links of the components in a copolymer chain $(\mathrm{PFD})_{N}(\mathrm{TFE})_{1-N}: N=0.65$ and 0.83 , correspondingly [10]. For the first time the problem of rigidity was considered in [10] quantitatively using methods of quantum chemistry and force field constants. The authors considered different models of the polymer chains consisting of 10 elementary links and established existence of the two spatial isomers with differ- ence between the two energy minima of $10.8 \mathrm{~kJ} / \mathrm{mol}$. Kinetic rigidity was determined using constants of the force field in the natural vibrational coordinates for the chain fragments PFD-PFD and PFD-TFE-PFD. Ratios between the fragments were different for AF2400 ("1") and AF1600 ("2"). The constant of the force field was found as the second derivative of potential energies $U_{1,2}$ of the model fragment over the angle $\varphi$ between the planes of the fragments (Eq. (1)). Thus, local rigidity is intermolecular characteristic of a polymer derived from sophisticated calculations. For substances "1" and "2"

$$
U_{1}^{\prime \prime}=\partial^{2} U_{1} / \partial^{2} \varphi>U_{2}^{\prime \prime}=\partial^{2} U_{2} / \partial^{2} \varphi
$$

Particularly, according to [10], the relation (1) means that the rigidity of AF2400 is higher than AF1600, as well as the expected free volumes. This is actually seen from the PAL experiments, which means further interconnection of the PAL and TSL data. We had tried to express this interconnection numerically considering the ratio $\alpha$ of the intensities of high-temperature and low-temperature components of the curve demonstrating a dependence of the integral TSL intensity on temperature. The way of calculation of the parameter $\alpha$ is shown in Fig. 3.

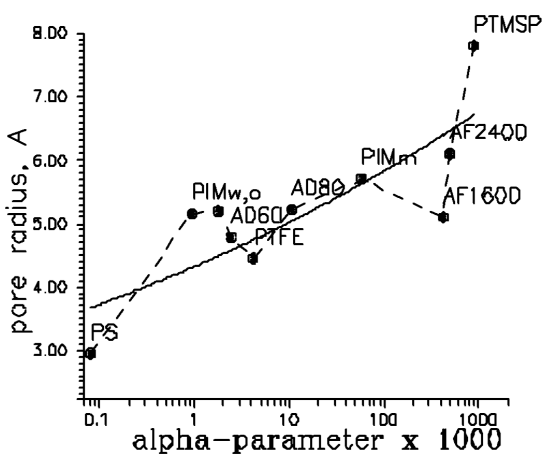

Fig. 4. Correlation of the pore effective radius (PAL experiments) and $\alpha$-parameter from the TSL experiments for a number of membrane materials (see the text, Sect. 4).

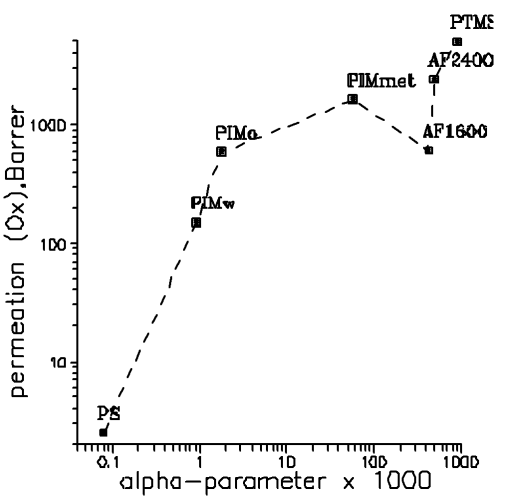

Fig. 5. Oxygen permeation for the polymer membrane materials from Fig. 4 as function of the parameter $\alpha$ found from the TSL measurements. 


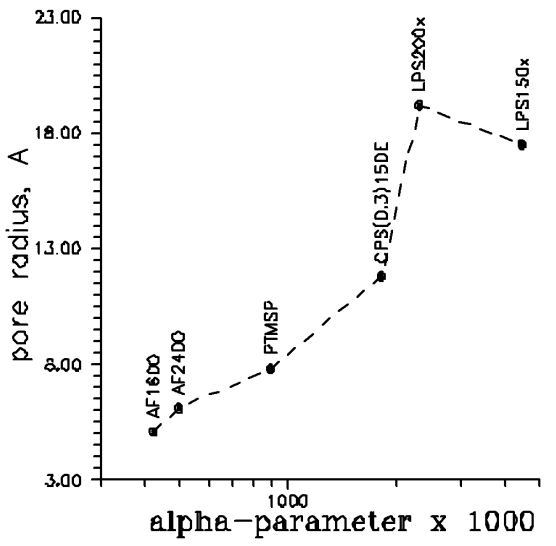

Fig. 6. The same dependence as in Fig. 4 for polymer sorbents CPS and LPS (see Sect. 4). Data for AF and PTMSP are given for comparison.

$\alpha=S_{\mathrm{HT}} / S_{\mathrm{LT}}$.

Conventional boundary for $S_{\mathrm{HT}}$ was taken at 200$250 \mathrm{~K}$. It is obvious that, in the TSL experiment, the $\alpha$-parameter has to increase along with rigidity of a polymer and, correspondingly, with the size of the free volume elements and permeation of the number of membrane materials. Figures $4-6$ illustrate this correlation on experiment.

Polymeric sorbents of the CPS type (Fig. 6) give similar but even stronger dependence of the effective radius of pores on the values of parameter $\alpha$, confirming in this way the suggested experimental approach.

\section{Mixed matrix membranes}

One of the contemporary methods of obtaining materials of high permeation and developed specific surface is preparation of mixed matrix compositions [5, 6], particularly MMMs with externally affected porosity. One of the components considered here is the polymer of intrinsic microporosity PIM $[1,11]$ already mentioned above. This polymer forms heterogeneous structures with zeolitic imidozolate framework ZIF with contents up to 48 vol.\% (Table from [6]).

TABLE

PAL characteristics, oxygen permeation $P\left(\mathrm{O}_{2}\right)$ and TSL $\alpha$-parameter for a number of composition materials (metal-organic frameworks). Data for polymeric membrane materials AF2400 and PTMSP are given for comparison with MMMs. Data in boldface are specially discussed in the text.

\begin{tabular}{c|c|c|c|c|c|c}
\hline \hline Polymer & $I_{3}[\%]$ & $\tau_{3}[\mathrm{~ns}]$ & $I_{4}[\%]$ & $\tau_{4}[\mathrm{~ns}]$ & $\begin{array}{c}P\left(\mathrm{O}_{2}\right) \\
{[\text { Barrer] }}\end{array}$ & $\alpha \times 10^{3}$ \\
\hline PHFP & $3.60 \pm 0.56$ & $1.69 \pm 0.21$ & $13.74 \pm 0.80$ & $\mathbf{4 . 8 4} \pm \mathbf{0 . 1 4}$ & $\mathbf{1 0 0}$ & $\mathbf{1 1 3}-\mathbf{1 3 0}$ \\
PHFP-SAPO 20 vol.\% & $1.27 \pm 0.32$ & $1.59 \pm 0.07$ & $8.91 \pm 0.38$ & $\mathbf{5 . 7 4} \pm \mathbf{0 . 1 3}$ & $\mathbf{1 6 0}$ & $\mathbf{4 8 7}$ \\
PIM (meth) & $8.21 \pm 0.81$ & $1.17 \pm 0.11$ & $17.81 \pm 0.26$ & $6.31 \pm 0.08$ & 1610 & 87.3 \\
PIM (orig) & $6.14 \pm 0.61$ & $2.13 \pm 0.30$ & $17.79 \pm 0.87$ & $\mathbf{5 . 8 1} \pm \mathbf{0 . 1 9}$ & $\mathbf{5 8 4}$ & $\mathbf{1 . 8}$ \\
ZIF & $17.68 \pm 0.46$ & $1.48 \pm 0.05$ & $19.99 \pm 0.27$ & $7.94 \pm 0.11$ & & 280 \\
PIM-ZIF 32 vol.\% & $24.72 \pm 0.37$ & $1.72 \pm 0.04$ & $13.73 \pm 0.38$ & $\mathbf{6 . 4 2} \pm \mathbf{0 . 1 2}$ & $\mathbf{8 7 0}$ & $\mathbf{4 1 0}$ \\
PIM-ZIF 48 vol.\% & $21.59 \pm 0.34$ & $1.82 \pm 0.04$ & $13.14 \pm 0.41$ & $\mathbf{6 . 4 3} \pm \mathbf{0 . 1 3}$ & $\mathbf{1 6 8 0}$ & $\mathbf{5 5 0}$ \\
AF-2400 & $2.6 \pm 0.3$ & $1.82 \pm 0.33$ & $13.7 \pm 0.25$ & $8.0 \pm 0.1$ & 2400 & 640 \\
PTMSP & $4.37 \pm 0.48$ & $2.68 \pm 0.45$ & $33.8 \pm 0.63$ & $11.8 \pm 0.22$ & 5000 & 890
\end{tabular}

The second example is a composition of poly(hexafluoro propylene) with another framework component SAPO (20 vol.\%). PAL and TSL measurements ( $\alpha$ parameter) were performed for the same samples. Figure 7 demonstrates intensive high temperature part of TSL curve $(T>250 \mathrm{~K})$ for the composition $\mathrm{PIM} / \mathrm{ZIF}$. This part is higher than for the elements of the composition. The data are combined with oxygen permeation measurements $P\left(\mathrm{O}_{2}\right)$, Barrer. Results in Table, as well as the whole previous data, confirm interconnection between the development of elementary free volumes and local rigidity. Particularly, they confirm the correctness of the theoretical approach [10]. However, for the PAL studies of elementary free volumes in heterogeneous systems, the data illustrate some limitations, too. Nonlocalized precursor of localized Ps has to find free volume before annihilation outside the pore (trapping before annihilation). For intrinsic pores of homogeneous polymers this is usually provided. But if we have wide spectrum of sizes, probability of localization by largest free volumes goes down. This is seen, for example, even on the case of homogeneous glassy polymer AF2400 (Fig. 8) [1], where results of PAL measurements of size distribution of elementary free volumes are compared with such distribution, calculated by the method of dynamic simulation. Positronium does not feel the largest (sometimes the most distant) pores. Meanwhile, namely the largest pores, on the edge of 


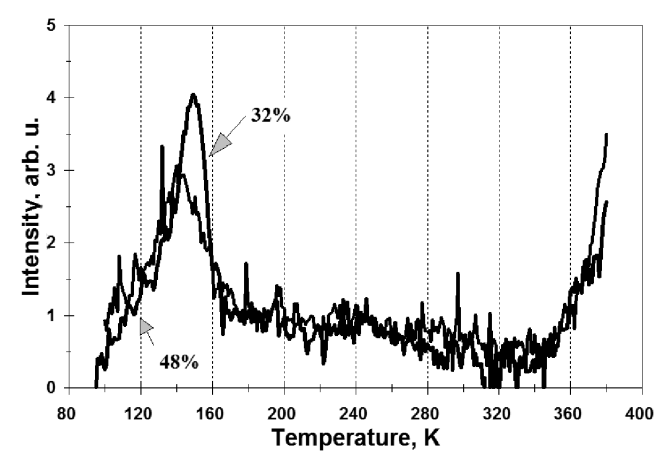

Fig. 7. TSL curve for the system PIM/ZIF with intensive high temperature component corresponding to high permeaion of the composition (see Table).

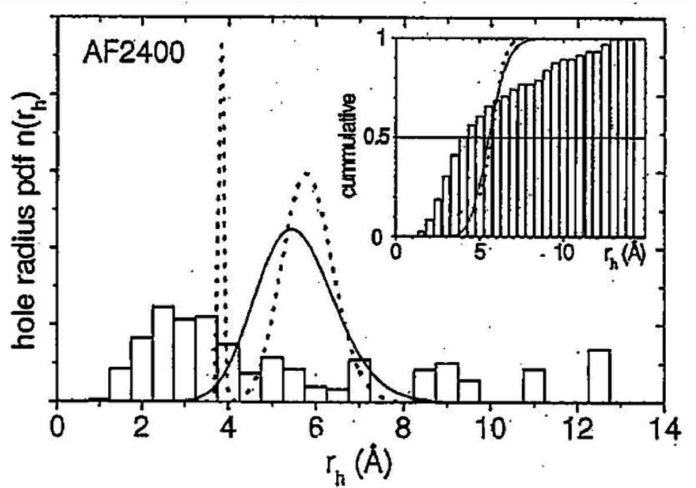

Fig. 8. Size distribution of elementary free volumes in AF2400 obtained by PAL and by mathematical modeling [1].

size distribution, may be responsible for the permeation and for the specific surface, i.e. the main properties of membranes and sorbents. The most clearly this effect reveals itself in the cases of heterogeneous composition materials, MMMs. The effect depends on the distances between the sites, modified by admixture. From Table, important, modified feature of the composition reveals itself by essential increase of permeation for oxygen $P\left(\mathrm{O}_{2}\right)$ compared to original polymer. Thus, in the case of PHFP-SAPO $P\left(\mathrm{O}_{2}\right)$ is changing from 100 to 160 Barrer and for PIM-ZIF from 584 to 1680 Barrer. At the same time, variations of the lifetimes are not so remarkable: the longest lifetimes $\tau_{4}$ obtained by the PATFIT analysis are changing from $4.84 \pm 0.14$ to $5.74 \pm 0.13 \mathrm{~ns}(4.7$ to $5.2 \AA$ ) and from $5.81 \pm 0.19$ to $6.43 \pm 0.13 \mathrm{~ns}(5.2$ to $5.5 \AA)$, correspondingly. We cannot be sure that namely these variations of the pore radius are responsible for the essential variations of the permeation. We may suggest that, due to heterogeneity of the mixtures, positronium in many cases fails to reach sites of the structure, mostly modified by the admixture, and responsible for variations of permeation. Low temperature gas sorption by the studied materials (BET measurements of the size distribution of elementary free volumes) could be helpful for final elucidation of the problem. Along with this, variations of the $\alpha$-parameter are very strong: from 113 to 487 in PHFPO/SAPO and from 584 to 1680 in PIM/ZIF. Thus, TSL method appears very helpful in the cases of PAL limitations. Comparing sensitivities of the PAL and TSL methods, we may suppose that this difference can be explained by the difference in the mobility of the precursors of the localized Ps (PAL) and mobility of free electron (TSL). We continue the studies of polymeric composition materials using combinations of PAL, TSL and the Brunauer-Emmett-Teller (BET) measurements.

\section{Conclusions}

1. The results obtained agree with the concept that storage of weakly bound electrons is responsible for the both, low temperature positronium formation and the effect of TSL. Both are related with the spur processes.

2. Specific shapes of the TSL curves for the components of compositions are helpful in the revealing of heterogeneities and transformations of the composition substances with unresolved positronium lifetimes.

3. Theoretical concept of local rigidity as a criterion of local free volume and gas permeation is proved on experiment by fine correlation of PAL data, permeation and TSL parameter $\alpha$ introduced by the authors.

4. Along with PAL measurements, TSL characteristics can be used as another instrument for revealing substances of high free volume and permeation, for the studies of polymer membrane materials and sorbents.

\section{References}

[1] M. Rudel, J. Krause, K. Ratzke, M. Faupel, Yu. Yampolskii, V. Shantarovich, G. Dlubek, Macromolecules 41, 788 (2008).

[2] V.P. Shantarovich, T. Suzuki, Y. Ito, K. Kondo, R.S. Yu, P.M. Budd, Yu.P. Yampolskii, S.S. Berdonosov, A.A. Eliseev, Phys. Status Solidi C 4, 3776 (2007).

[3] V.P. Shantarovich, J. Polym. Sci., Part B, Polym. Phys. 46, 2485 (2008).

[4] Principles and Applications of Positron and Positronium Chemistry, Eds. Y.C. Jean, P.E. Mallon, D.M. Schrader, World Sci., New Jersey 2003, p. 167.

[5] P. Winberg, K. Desitter, C. Datremont, S. Mullens, I.F.J. Vankelecom, F.H.J. Maurer, Macromolecules 38, 3776 (2005). 
[6] A.F. Bushell, M.P. Attfield, C.R. Mason, P.M. Budd, Yu. Yampolskii, L. Starannikova, A. Rebrov, F. Bazarelli, P. Bernardo, J.C. Jason, M. Lanc, K. Friess, V. Shantarovich, V. Gustov, V. Isaeva, J. Membr. Sci. 427, 48 (2013).

[7] V.P. Shantarovich, V.W. Gustov, T.I. Medintseva, A.V. Polyakova, E.V. Belousova, M.K. Filimonov, E.V. Prut, Doklady Phys. Chem. 441, 237 (2011); Mater. Sci. Forum 666, 81 (2011).

[8] S. Claes, P. Vanderzande, S. Mullens, M.K. Van Bael, F.H.J. Maurer, Macromolecules 44, 2766 (2011).

[9] V. Shantarovich, V. Gustov, A. Polyakova, E. Belousova, M. Filimonov, Yu. Yampolskii, Phys. Status Solidi C 6, 2387 (2009).

[10] A.V. Tokarev, G.N. Bondarenko, Yu.P. Yampolskii, Vysokomolek. Soed. (Polymer Sci., Russ.) 49, 1510 (2007).

[11] P.M. Budd, K.J. Msaib, C.S. Tattershall, B.S. Ghanem, K.J. Raynolds, N.B. McKeown, D. Fritsch, J. Membr. Sci. 251, 263 (2005).

[12] O.E. Mogensen, Positron Annihilation in Chemistry, Springer-Verlag, Berlin 1995, p. 66.

[13] T. Hirade, F.H.J. Maurer, M.E. Eldrup, Radiat. Phys. Chem. 58, 465 (2000).
[14] V.P. Shantarovich, T. Hirade, I.B. Kevdina, V.W. Gustov, E.F. Oleinik, Acta Phys. Pol. A 99, 497 (2001).

[15] Y. Ito, T. Hirade, E. Hamada, T. Suzuki, Y. Ito, Acta Phys. Pol. A 95, 433 (1999).

[16] V.G. Nikolskii, High Energy Chem., Russ. 2, 271 (1968); Pure Appl. Chem. 54, 493 (1982).

[17] V.P. Shantarovich, R.S. Yu, Ya. Kino, Ya. Hama, V.W. Gustov, High Energy Chem. (Russ.) 45, 1 (2011).

[18] D. Hofmann, M. Heuchel, Yu. Yampolskii, V. Khotimskii, V. Shantarovich, Macromolecules 35, 2129 (2002).

[19] V.P. Shantarovich, T. Suzuki, C. He, V.A. Davankov, A.V. Pastukhov, M.P Tsyurupa, K. Kondo, Y. Ito, Macromolecules 35, 9723 (2002).

[20] D. Hofmann, M. Entrialgo Costano, A. Lerbert, M. Heuchel, Y. Yampolskii, Macromolecules 36, 8528 (2003). 